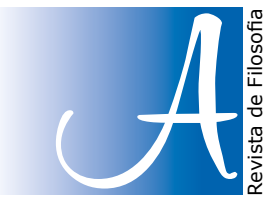

ARGUMENTOS

\section{Artigos}

Hércules de Araujo Feitosa*, Marcelo Reicher Soares**,

Cristiane Alexandra Lázaro***

\title{
Lógica deôntica básica e filtros
}

\author{
Basic logic deontic and filters
}

\section{RESUMO}

A lógica deôntica é um ramo da lógica simbólica interessada em noções como obrigatório, permitido, proibido e outras similares. Uma apresentação da Lógica Deôntica Padrão, conhecida como a lógica modal KD, tem uma aparência que lembra a estrutura matemática dos filtros. Mostramos que, de fato, a classe de filtros próprios em álgebras booleanas é um modelo adequado para KD.

Palavras-chave: Lógica deôntica, Filtros, Lógicas Modais, Modelos para lógicas modais.

\section{ABSTRACT}

Deontic logic is a branch of symbolic logic interested in notions as obligatory, permissible, forbidden, and similar ones. One presentation of Standard Deontic Logic, well known as the modal logic KD, has an appearance that remember the mathematical structure of filters. So we show that indeed the class of proper filters on Boolean algebras are adequate model for KD.

Keywords: Deontic logic. Filters. Modal logics. Models for modal logics.

\section{Introdução}

O principal objetivo deste artigo é mostrar que o conceito usual e simples de filtro próprio constitui um modelo para a lógica deôntica básica, também conhecida na literatura sobre lógicas modais como o sistema KD.

Uma vez que o tema está inserido no contexto das lógicas modais, iniciamos com uma visão bastante breve das lógicas modais. Em seguida, como um caso particular de lógica modal, apresentamos a lógica deôntica básica, ou o sistema

\footnotetext{
* Doutor em Lógica e Filosofia da Ciência - UNICAMP. https://orcid.org/0000-0003-0023-4192.

** Doutor em Matemética - USP https://orcid.org/0000-0002-1996-5350.

*** Doutora em Matemática - UNICAMP. https://orcid.org/0000-0002-0260-6976.
} 
modal KD, conforme podemos encontrar nos muitos artigos e livros sobre lógicas modais e lógicas deônticas, em particular.

Uma apresentação em versão axiomática, encontrado no livro (CHELLAS, 1980), mais alguma experiência no tratamento do conceito matemático de filtro, nos permitiu observarmos que os filtros poderiam ser vistos como modelos para a lógica KD. Procuramos e não encontramos a vinculação entre estes dois tópicos na literatura sobre lógicas modais e deônticas.

Assim, como nossa contribuição original, mostramos que classe de filtros próprios, que chamamos de D-espaços, são modelos adequados para o sistema KD.

\section{Lógicas Modais}

A Lógica Modal surgiu no início do século XX e tem, entre outros, a atribuição de qualificar a verdade de um julgamento, ou seja, o modo do julgamento. Trabalhos essenciais da primeira metade daquele século destacaram o comportamento formal de expressões como 'é necessário que' e 'é possível que', usando uma linguagem proposicional formal com dois operadores modais para necessário e possível.

É usual utilizarmos os símbolos $\square$ e $\diamond$ para essas duas noções, necessário e possível, respectivamente.

O tema 'lógica modal', no entanto, é mais amplo que a investigação desses dois termos aléticos e suas correlações. $\mathrm{Na}$ verdade ele caracteriza uma família de sistemas lógicos, com várias distintas modalidades.

Essa familia inclui lógicas temporais, lógicas epistêmicas, lógicas aléticas, lógicas doxásticas, entre outras, e como particularmente de nosso interesse, as lógicas deônticas.

Lógicas deônticas enfatizam expressões como 'é obrigatório que', 'é permitido que', e 'é proíbido que'.

Em textos introdutórios sobre lógicas modais, aparece uma família modal construída a partir de uma lógica fraca chamada e denotada por $\mathbf{K}$, em honra à Saul Kripke, que na década de 1950 introduziu os modelos de Kripke para essa família de lógicas.

Essa família tem sua linguagem construída a partir do conjunto $L=\{\neg$, $\wedge$, $\vee, \rightarrow, \square\}$ tal que os quatro primeiros operadores são os clássicos e o último é o operador modal para necessário.

Usualmente, o operador para possibilidade é definido a partir de $\square$ por:

$$
\diamond \varphi=_{d f} \neg \square \neg \varphi .
$$

O sistema $\mathrm{K}$ é obtido por adição dos seguintes dois princípios à lógica proposicional clássica (LPC):

Regra Nec: $\vdash \varphi / \vdash \square \varphi$

Axioma K: $\square(\varphi \rightarrow \psi) \rightarrow(\square \varphi \rightarrow \square \psi)$.

O axioma $\mathrm{K}$ é também conhecido como Axioma de Distributividade e a Regra Nec, de necessitação, diz que todo teorema é necessário.

Nós trataremos com a Lógica Deontica, a Lógica Deôntica Standard (KD), que é obtida por introdução, no sistema $\mathbf{K}$, do símbolo primitivo 0 para 'é obrigatório 
que', no lugar de $\square$. Então, a partir do operador 0, podemos definir o operador $\mathrm{P}$, para 'é permitido que', e F, para 'é proibido que', como veremos a seguir.

A lógica KD admite ainda o axioma deôntico fundamental D: $0 \varphi \rightarrow \mathrm{P} \varphi$, que diz que se $\varphi$ é obrigatório, então $\varphi$ é permitido.

Dessa forma, obtemos um particular e interessante sistema lógico modal, como apresentado em (CHELLAS, 1980).

Considerando a lógica KD apresentada na próxima seção, nós introduzimos o conceito de $D$ - espaços, que são estruturas matemáticas planejadas e obtidas a partir de filtros, destinadas à serem modelos para KD.

Finalmente nós mostramos a adequação desse modelo para KD.

\section{O sistema deôntico básico}

A Lógica Deôntica Básica, conhecida como o sistema KD, é gerada pela inclusão do axioma $\mathrm{D}$ na lógica modal mais elementar na hierarquia das lógicas modais normais, que conta apenas com o axioma $\mathrm{K}$ e a regra de necessitação Nec.

Axioma D: $0 \varphi \rightarrow \mathrm{P} \varphi$.

Nas Lógicas Deônticas utilizamos a seguinte interpretação: 0 para 'é obrigatório que', $P$ para 'é permitido que' e $F$ para 'é proibido que'.

Logo, o axioma $\mathrm{D}, 0 \varphi \rightarrow P \varphi$, nos diz que, se a fórmula é obrigatória, então é permitida.

Se tomamos o operador de obrigação 0 como básico, ou padrão, podemos definir os operadores de permissão e proibição, da seguinte forma:

$$
\begin{aligned}
& P \varphi={ }_{d f} \neg 0 \neg \varphi \\
& F \varphi={ }_{d f} \neg 0 \neg \varphi .
\end{aligned}
$$

Apresentaremos três diferentes representações, porém equivalentes, para a lógica KD. A última é menos usual, porém é providencial para o modelo que devemos lhe atribuir.

Consideremos a linguagem proposicional $L=\{\neg, \wedge, \vee, \rightarrow, 0\}$, com $\operatorname{Var}(\mathrm{KD})=\left\{p_{1}, p_{2}, p_{3}, \ldots\right\}$ o conjunto de variáveis proposicionais, e os operadores $P$ e $F$ definidos como acima. For(KD) denotará o conjunto de fórmulas de KD.

Como estamos apresentando sistemas axiomáticos dedutivos, consideramos os conceitos usuais de dedução e demonstração.

Definição 2.1 Se $\Gamma \subseteq$ For(KD) e Ax denota um conjunto de axiomas de KD, então $C(\Gamma)=\{\psi: \Gamma \cup A x \vdash \psi\}$ é o conjunto de consequências de $\Gamma$.

Definição 2.2 Dizemos que $\psi$ é derivável em KD ou $\psi$ é um teorema de KD, quando $\psi \in C(\varnothing)$.

Definição 2.3 Uma teoria de KD é um conjunto $\Delta \subseteq$ For $(\mathrm{KD})$, tal que $C(\Delta)=\Delta$.

Quando $\Delta=\emptyset$, temos os teoremas de KD, isto é,

$$
\psi \in C(\emptyset) \Leftrightarrow \vdash \psi \text {. }
$$

O sistema lógico KD pode ser caracterizado por alguns dos seguintes sistemas dedutivos:

Por $\mathrm{KD}_{1}$, como em (CARNIELLI, 2001, p. 16-18). 
(LPC) $\varphi$, se $\varphi$ é uma tautologia

(K) $\mathrm{O}(\varphi \rightarrow \psi) \rightarrow(\mathrm{O} \varphi \rightarrow \mathrm{O} \psi)$

(D) $0 \varphi \rightarrow P \varphi$

(MP) $\varphi \rightarrow \psi, \varphi / \psi$

$(\mathrm{Nec}) \vdash \varphi / \vdash \mathrm{O} \varphi$.

Porém, em (CHELLAS, 1980), encontramos mais duas apresentações:

$\mathrm{KD}_{2}$ :

(LPC) $\varphi$, se $\varphi$ é uma tautologia

$\left(\mathrm{OD}^{\star}\right) \neg(\mathrm{O} \varphi \wedge \mathrm{O} \neg \varphi)$

(MP) $\varphi \rightarrow \psi, \varphi / \psi$

$(\mathbf{R O K}) \vdash\left(\varphi_{1} \wedge \ldots \wedge \varphi_{n}\right) \rightarrow \psi / \vdash\left(\mathrm{O} \varphi_{1} \wedge \ldots \wedge \mathrm{O} \varphi_{n}\right) \rightarrow 0 \psi$, para $\mathrm{n} \geq 0$.

$\mathrm{KD}_{3}$ :

(LPC) $\varphi$, se $\varphi$ é uma tautologia

(OC) $(\mathrm{O} \varphi \wedge \mathrm{O} \psi) \rightarrow \mathrm{O}(\varphi \wedge \psi)$

(ON) OT

(OD) $\neg 0 \perp$

(MP) $\varphi \rightarrow \psi, \varphi / \psi$

(ROM) $\vdash \varphi \rightarrow \psi / \vdash O \varphi \rightarrow 0 \psi$.

Proposição 2.4 Os sistemas $\mathrm{KD}_{2}$ e $\mathrm{KD}_{3}$ são dedutivamente equivalentes.

Demonstração: Primeiramente, vamos obter $\mathrm{OD}^{*}$ e $\mathrm{ROK}$ em $\mathrm{KD}_{3}$.

Por $\mathrm{OC}$ temos que $(\mathrm{O} \varphi \wedge \mathrm{O} \neg \varphi) \rightarrow \mathrm{O}(\varphi \wedge \neg \varphi) \Leftrightarrow(\mathrm{O} \varphi \wedge \mathrm{O} \neg \varphi) \rightarrow 0 \perp$. A partir daí, utilizando $\mathrm{OD}$, segue que $\neg(\mathrm{O} \varphi \wedge \mathrm{O} \neg \varphi)$, ou seja, vale OD*.

Como $(\varphi \wedge \psi) \rightarrow \varphi \mathrm{e} \rightarrow(\varphi \wedge \psi) \rightarrow \psi$, então, por ROM obtemos que, $\mathrm{O}(\varphi \wedge \psi) \rightarrow$ $\mathrm{O} \varphi$ e $\mathrm{O}(\varphi \wedge \psi) \rightarrow \mathrm{O} \psi$ e, então, que $\mathrm{O}(\varphi \wedge \psi) \rightarrow(\mathrm{O} \varphi \wedge \mathrm{O} \psi)$. Agora, compondo com OC, temos que $\mathrm{O}(\varphi \wedge \psi) \leftrightarrow(\mathrm{O} \varphi \wedge \mathrm{O} \psi)$, que pode ser estendida para:

$$
\mathrm{O}\left(\varphi_{1} \wedge \ldots \wedge \varphi_{n}\right) \leftrightarrow\left(\mathrm{O} \varphi_{1} \wedge \ldots \wedge \mathrm{O} \varphi_{n}\right) .[1]
$$

Deste modo, se tomarmos $\vdash\left(\varphi_{1} \wedge . . \wedge \varphi_{n}\right) \rightarrow \psi$, por $\operatorname{ROM}$ obtemos $\vdash\left(\varphi_{1} \wedge \ldots\right.$ $\left.\wedge \varphi_{n}\right) \rightarrow 0 \psi$. Logo, com a equivalência de [1] temos que $\vdash\left(0 \varphi_{1} \wedge . . \wedge 0 \varphi_{n}\right) \rightarrow 0 \psi$, ou seja, temos ROK em $\mathrm{KD}_{3}$.

Por outro lado, precisamos obter OC, ON, OD e ROM em $\mathrm{KD}_{2}$.

De ROK, considerando $n=0$, temos $\vdash \varphi / \vdash 0 \varphi$. Como $\vdash T$, então $\vdash 0 T$, ou seja, temos ON.

Novamente por ROK, agora $\operatorname{com} n=1$, temos ROM.

De $\vdash(\varphi \wedge \psi) \rightarrow(\varphi \wedge \psi)$ e ROK, temos OC.

Finalmente, por OD*, temos $\neg \mathrm{O} \perp \vee \neg \mathrm{O} \neg \perp \Leftrightarrow \neg \mathrm{O} \perp \vee \neg \mathrm{OT}$. Considerando que em $\mathrm{KD}_{3}$ vale $\mathrm{ON}$, temos então $\neg 0 \perp$. -

Proposição 2.5 Os sistemas $\mathrm{KD}_{1}$ e $\mathrm{KD}_{2}$ são dedutivamente equivalentes.

Demonstração: Vamos verificar primeiramente que os axiomas e regras de $\mathrm{KD}_{1}$ são obtidos em $\mathrm{KD}_{2}$.

Por ROK, $\operatorname{com} n=0$, temos Nec.

De OD*, podemos obter $\mathrm{D}$, da seguinte forma:

$$
\neg(\mathrm{O} \varphi \wedge \mathrm{O} \neg \varphi) \Leftrightarrow \neg \mathrm{O} \varphi \vee \neg \mathrm{O} \neg \varphi \Leftrightarrow \neg 0 \varphi \vee P \varphi \Leftrightarrow 0 \varphi \rightarrow P \varphi .
$$


Finalmente, considerando a tautologia $((\varphi \rightarrow \psi) \wedge \varphi) \rightarrow \psi$, usando ROK temos que $(\mathrm{O}(\varphi \rightarrow \psi) \wedge \mathrm{O} \varphi) \rightarrow \mathrm{O} \psi$. Agora, através de LPC, temos que $\mathrm{O}(\varphi \rightarrow \psi) \rightarrow(\mathrm{O} \varphi \rightarrow \mathrm{O} \psi)$.

Agora, vamos mostrar que os axiomas e regras de $\mathrm{KD}_{2}$ são obtidos em $\mathrm{KD}_{1}$. Como acima, $O D^{*}$ e D são equivalentes.

Falta mostrarmos ROK em KD .

Se $\vdash\left(\varphi_{1} \wedge \ldots \wedge \varphi_{n}\right) \rightarrow \psi /$ por Nec segue que $\vdash O\left(\left(\varphi_{1} \wedge \ldots \wedge \varphi_{n}\right) \rightarrow \psi\right)$ e, utilizando $\mathbf{K}$, temos que $\vdash \mathrm{O}\left(\varphi_{1} \wedge . . . \wedge \varphi_{n}\right) \rightarrow 0 \psi$. Logo, através da equivalência [1], temos que $\vdash\left(0 \varphi_{1} \wedge \ldots \wedge O \varphi_{n}\right) \rightarrow O \psi$.

\section{Filtros}

Nesta seção, apresentamos o conceito de filtros. Filtros e ultrafiltros são estruturas bastante frequentes no contexto da Lógica. São relativamente simples e as estruturas de filtro próprios são planejadas, nestas notas, para serem os modelos da lógica KD.

Definição 3.1 Um filtro sobre um conjunto $E$ é uma coleção não vazia $\mathcal{F} \subseteq \mathcal{P}(E)$ tal que:

(i) se $A, B \in \mathcal{F}$, então $A \cap B \in \mathcal{F}$

(ii) se $A \in \mathcal{F}$ e $A \subseteq B$, então $B \in \mathcal{F}$.

Como $\mathcal{F}$ é não vazio, então sempre $E \in \mathcal{F}$.

Para um conjunto $E$ qualquer, as coleções $P(E)$ e $\{E\}$ são casos triviais de filtros.

Proposição 3.2 Toda intersecção de filtros sobre E é ainda um filtro sobre $E$.

Demonstração: Seja $\mathcal{F}_{i}$, com $i \in \Lambda$, uma familia de filtros sobre E. Então, $\cap \mathcal{F}_{i}$ é um filtro pois: (i) se $A, B \in \cap \mathcal{F}_{i}$, então para cada $i \in \Lambda$, tem-se que $A, B \in \mathcal{F}_{i}$ e posto que $\mathcal{F}_{i}$ é filtro, segue que $A \cap B \in \mathcal{F}_{i}$ e, portanto, $A \cap B \in \cap \mathcal{F}_{i}$; (ii) se $A \in \cap \mathcal{F}_{i}$ e $A \subseteq B$, então $A \in \mathcal{F}_{i}$, para cada $i \in \Lambda$, e como $\mathcal{F}_{i}$ é filtro, segue que $B \in \mathcal{F}_{i}$ e, portanto, $B \in \cap \mathcal{F}_{i}$.

Definição 3.3 Um filtro sobre E é próprio se $\mathcal{F}$ é tal que $\mathcal{F} \neq \mathcal{P}(E)$.

Se $\mathcal{F}$ é próprio, então $\emptyset \notin \mathcal{F}$.

Uma intersecção de filtros próprios é um filtro próprio, pois o conjunto $\emptyset$ não pertence a qualquer membro da coleção.

Definição $3.4 \bigcirc$ filtro $\mathcal{F}$ é livre se $\cap \mathcal{F}=\emptyset$.

Exemplo 3.5 Para cada subconjunto não vazio $A \subseteq E$, o conjunto $[A]=\{C \subseteq E$ : $A \subseteq C$ \} é um filtro sobre $E$.

Este filtro é denominado de filtro principal gerado por $A$ e é o menor filtro sobre $E$ que contém $A$.

Se $A=\{x\}$, o filtro principal gerado por $A$ é o conjunto $[A]=\{C \subseteq E: x \in C\}$.

Exemplo 3.6 Se E é infinito, então um subconjunto $A \subseteq E$ é cofinito se seu complemento em $E, A^{C}$, é finito.

A família de todos os subconjuntos cofinitos de $E$ é um filtro sobre $E$. Este filtro é chamado de filtro de Fréchet sobre $E$.

Cada filtro de Fréchet é um exemplo de filtro livre. 
Proposição 3.7 Se o filtro $\mathcal{F}$ é livre, então ele é não principal.

Demonstração: Seja $\mathcal{F}$ um filtro sobre $E$. Se $\mathcal{F}$ é principal, então $\mathcal{F}=[A], A \neq \emptyset$ e $A \in \mathcal{F}$. Assim, $\cap \mathcal{F}=A$ e, portanto $\mathcal{F}$ não é livre.

Proposição 3.8 Se E é finito, então todo filtro próprio sobre E é principal.

Demonstração: Seja $\mathcal{F}$ um filtro próprio sobre o conjunto finito $E$. Assim, $\mathcal{P}(E)$ também é finito e $B=\cap \mathcal{F} \in \mathcal{F}$. Logo $\mathcal{F}=[B]$ e como $\mathcal{F}$ é próprio, então $B \neq \perp$. Portanto, $\mathcal{F}$ é principal.

Proposição 3.9 Se $\mathcal{F}$ é um filtro sobre $E$, então ele é livre se, e somente se, ele contém o filtro de Frechet sobre E.

Demonstração: Se $\mathcal{F}$ é livre, então a intersecção de todo conjunto em $\mathcal{F}$ é vazia. Assim, para cada $x \in E$ existe um conjunto $B_{x} \in \mathcal{F}$ tal que $x \notin B_{X}$, e $B_{X} \subseteq E-\{x\}$ e $E-\{x\} \in \mathcal{F}$.

Como cada conjunto cofinito é uma intersecção de conjuntos do tipo $E-\{X\}$, então todos conjuntos cofinitos estão em $\mathcal{F}$. Logo, $\mathcal{F}$ contém o filtro de Frechet sobre $E$.

Por outro lado, se $\mathcal{F}_{f}$ é o filtro de Frechet sobre $E$ e para um filtro qualquer $\mathcal{F}$, temos que $\mathcal{F}_{f} \subseteq \mathcal{F}$. Então $\cap \mathcal{F} \subseteq \mathcal{F}_{f}=\emptyset$, pois $\mathcal{F}_{f}$ é livre. Logo, $\mathcal{F}$ é livre também.

Definição 3.10 Uma família A de subconjuntos de E tem a proprieade da intersecção finita (fip) se toda família finita de $A$ tem intersecção não vazia.

Os filtros próprios têm a propriedade da intersecção finita, pois se:

$$
A_{1}, \ldots, A_{n} \in \mathcal{F} \text {, então } \cap_{i=1}^{n} A_{i} \in \mathcal{F} \text { e } \cap_{i=1}^{n} A_{i} \neq \emptyset \text {. }
$$

Proposição 3.11 Se $A \subseteq \mathcal{P}(E)$ tem a fip, então existe um filtro minimal $\mathcal{F}_{A}$ que contém A.

Demonstração: Seja $\mathcal{F}_{A}=\{B \subseteq E: B$ inclui todas as interseções finitas de $A\}$. Deste modo, $B$ é fechado para intersecções finitas e para super conjuntos; e $\mathcal{F}_{A}$ é minimal posto que não existe filtro contido propriamente nele. -

Definição $3.12 \bigcirc$ filtro $\mathcal{F}_{A}$ é o filtro gerado por $A$.

Corolário 3.13 Seja $A \subseteq \mathcal{P}(E)$. Então A está incluso em um filtro sobre E se, e somente se, A tem a fip.

Nós podemos definir um D-espaço do seguinte modo.

Definição $3.14 U m$-espaço é um par $(E, \mathcal{F})$ em que $E$ é um conjunto não vazio e $\mathcal{F}$ é um filtro próprio sobre $E$

\section{D-espaços como modelos para KD}

Agora vemos como que os $\mathrm{D}$-espaços se configuram como modelos para a lógica deôntica básica, KD.

Seja $\mathcal{B}$ a álgebra Booleana de conjuntos $\mathcal{B}=\left(P(E), \emptyset, E, \cap, \cup,{ }^{C}\right)$, que é um modelo para a LPC.

Definição 4.1 Uma valoração Booleana é uma função ṽ :Var(LPC) $\rightarrow \mathcal{B}$ que aplica cada variável da LPC em um conjunto $A \subseteq E$. 
A valoração $\tilde{V}$ deve ser estendida para todo o conjunto de fórmulas For(LPC), pela função $v:$ For $($ LPC $) \rightarrow \mathcal{B}$ de modo que, para toda $p \in \operatorname{Var}($ LPC $)$ e todas $\varphi, \psi \in$ For(LPC) sejam válidas:

$$
\begin{aligned}
& v(p)=\tilde{v}(p) \\
& v(\neg \varphi)=[v(\varphi)]^{C} \\
& v(\varphi \wedge \psi)=v(\varphi) \cap v(\psi) \\
& v(\varphi \vee \psi)=v(\varphi) \cup v(\psi) .
\end{aligned}
$$

No caso acima dizemos que $v$ é uma valoração em $\mathcal{B}$.

Como usualmente, os símbolos de operadores do lado esquerdo da igualdade representam operadores lógicos, enquanto que os símbolos do lado direito representam operadores conjuntistas.

Definição 4.2 Uma valoração $v:$ For (LPC) $\rightarrow \mathcal{B}$ é um modelo para um conjunto $\Gamma \subseteq$ For $(\mathrm{LPC})$ se $=v(\varphi)=E$, para cada fórmula $\varphi \in \Gamma$. Dizemos então que $(\mathcal{B}, v)$ é um modelo para $\Gamma$.

Agora, estendemos o modelo $\mathcal{B}$ para adaptá-lo para a lógica KD. Para isso, consideraremos um $\mathrm{D}$-espaço $(\mathcal{B}, \mathcal{F})$ em $\mathcal{B}$ e denotamos esse fato escrevendo $\mathcal{B}^{\mathcal{F}}$. Decorre que teremos agora uma função $v:$ For $(\mathrm{KD}) \rightarrow \mathcal{B}^{\mathcal{F}}$ tal que, para a parte Booleana de For(KD), a interpretação é a usual em $\mathcal{B}$. Já para fórmulas do tipo $0 \psi \in$ For(KD) descrevemos seu comportamento na sequência.

Definição $4.3 A$ validade de uma fórmula de $\varphi \in F$ For $(\mathrm{KD})$ em $\mathcal{B}^{\mathcal{F}}$, denotada por $\mathcal{B}^{\mathcal{F}} \vDash \varphi$, é definida indutivamente por:

(i) se o operador principal de $\varphi$ é Booleano, então:

$$
\mathcal{B}^{\mathcal{F}} \vDash \varphi \Leftrightarrow \mathcal{B} \vDash \varphi \text {; }
$$

(ii) se $\varphi=0 \psi$, então:

$$
\mathcal{B}^{\mathcal{F}} \vDash \varphi \Leftrightarrow v(\psi) \in \mathcal{F} \text {, qualquer que seja a valoração } v \text { em } \mathcal{B}^{\mathcal{F}} \text {. }
$$

Lembramos que $\mathcal{B} \vDash \varphi$, significa $v(\varphi)=E$, para qualquer valoração $v$ em $\mathcal{B}$.

Definição 4.4 Dado um conjunto $\Gamma \subseteq F$ or $(\mathrm{KD})$, dizemos que o $D$-espaço $\mathcal{B}^{\mathcal{F}}$ é um modelo para $\Gamma$ se tivermos que $\mathcal{B}^{\mathcal{F}} \vDash \varphi$, para toda $\varphi \in \Gamma$.

Neste caso dizemos também que $\mathcal{B}^{\mathcal{F}}$ satisfaz $\Gamma$ ou ainda que cada $\varphi \in \Gamma$ é verdadeira em $\mathcal{B}^{\mathcal{F}}$.

Definição 4.5 Uma fórmula $\varphi \in$ For(KD) é dita válida se é verdadeira em todo $D$-espaço $\mathcal{B}^{\mathcal{F}}$.

Proposição 4.6 Para qualquer valoração $v$ em $\mathcal{B}^{\mathcal{F}}$ temos que $v(\varphi) \subseteq v(\psi) \Leftrightarrow$ $\vdash \varphi \rightarrow \psi$.

Demonstração: Temos que $v(\varphi) \subseteq v(\psi) \Leftrightarrow v(\varphi) \cup v(\psi)=v(\psi) \Leftrightarrow v(\varphi \vee \psi)=$ $v(\psi) \Leftrightarrow \Leftrightarrow \vdash \varphi \vee \psi \leftrightarrow \psi \Leftrightarrow \vdash \varphi \rightarrow \psi$

Teorema 4.7 (Correção) Os D-espaços $\mathcal{B}^{\mathcal{F}}$ são modelos corretos para a lógica KD.

Demonstração: Dado um D-espaço $\mathcal{B}^{\mathcal{F}}$, e uma valoração qualquer $v$ em $\mathcal{B}^{\mathcal{F}}$, precisamos mostrar que os axiomas ON, OC, OD são válidos e a regra Rom preserva a validade: 
ON: $\mathcal{B}^{\mathcal{F}} \vDash O T \Leftrightarrow V(T)=E \in \mathcal{F}$.

OD: $\mathcal{B}^{\mathcal{F}} \vDash \neg \mathrm{O} \perp \Leftrightarrow \mathcal{B}^{\mathcal{F}} \not \models \mathrm{O} \perp \Leftrightarrow$ para aluma valoração $v$ temos $v(\perp)=\emptyset \notin \mathcal{F}$.

OC: $\mathcal{B}^{\mathcal{F}} \vDash 0 \varphi \wedge 0 \psi \Leftrightarrow \mathcal{B}^{\mathcal{F}} \vDash 0 \varphi$ e $\mathcal{B}^{\mathcal{F}} \vDash 0 \psi \Leftrightarrow v(\varphi) \in \mathcal{F}$ e $v(\psi) \in \mathcal{F}$. Então, como necessariamente $v(\varphi) \cap v(\psi) \in \mathcal{F}$ segue que $\mathcal{B}^{\mathcal{F}} \vDash O(\varphi \wedge \psi)$.

Rom: Se $\vdash \varphi \rightarrow \psi$ então segue, da Proposição 4.6, que, $v(\varphi) \subseteq v(\psi)$. Agora, se $\mathcal{B}^{\mathcal{F}} \vDash 0 \varphi$, então $v(\varphi) \in \mathcal{F}$. Como $v(\varphi) \subseteq v(\psi)$, decorre que $v(\psi) \in \mathcal{F}$. Logo, $\vdash 0 \varphi \rightarrow 0 \psi$.

O corolário seguinte é conhecido como a Correção Forte. Embora em muitos casos de lógicas modais, devido a particularidades dedutivas de tais sistemas, este desenvolvimento se encerra na Correção Fraca, como no teorema anterior, para KD, a versão forte é bastante simples.

Corolário 4.8 Se $\Gamma \vdash \varphi$, então $\Gamma \vDash \varphi$.

Demonstração: Suponhamos que $\Gamma \vdash \varphi$, e seja $\mathcal{B}^{\mathcal{F}}$ um modelo tal que $\mathcal{B}^{\mathcal{F}} \vDash \Gamma$.

A demonstração é por indução sobre o comprimento da dedução $\Gamma \vdash \varphi$.

Se $n=1$, então $\varphi$ é um axioma (teorema) ou $\varphi$ pertence $\Gamma$.

Se é um axioma, o resultado segue do Teorema 4.7. Se $\varphi$ pertence a $\Gamma$, naturalmente $\Gamma \vDash \varphi$.

Agora, para $n>1$, temos que $\varphi$ foi obtida pela regra MP ou pela regra Rom.

No caso da MP, o resultado é usual. E no caso da Rom, o resultado segue exatamente como no Teorema 4.7. -

Por outro lado, precisamos mostrar a recíproca deste corolário, o Teorema da Completude. De um modo geral, a completude forte pode ser delicada para os sistemas modais, devido principalmente à regra Nec, mas para KD é usual como pode ser visto em (CHAGROV, ZAKHARYASCHEV, Cap. 3 e 5, 1997), para os modelos de Kripke.

Definição 4.9 Um conjunto de fórmulas $\Delta$ é consistente maximal se ele é consistente, e nenhuma extensão própria de $\Delta$ é consistente.

Proposição 4.10 $\Gamma \vdash \psi \Leftrightarrow \Gamma \cup\{\neg \psi\}$ é inconsistente.

Demonstração: (FEITOSA, PAULOVICH, 2005, p. 81). -

Teorema 4.11 Se $\Gamma$ é consistente maximal, então para toda fórmula $\psi$ de For (TK) ou $\psi \in \Gamma$ ou $\neg \psi \in \Gamma$.

Demonstração: (FEITOSA, PAULOVICH, 2005, p. 81). -

Proposição 4.12 Se $\Gamma$ é consistente maximal e $\Gamma \vdash \psi$, então $\psi \in \Gamma$.

Demonstração: (FEITOSA, PAULOVICH, 2005, p. 81).

Teorema 4.13 (Lindenbaum) Todo conjunto consistente de fórmulas $\Gamma$ pode ser estendido a um conjunto consistente maximal $\Delta$.

Demonstração: A demonstração é usual e pode ser encontrada em (FITTING, MENDELSOHN, 1998, p. 76).

Teorema 4.14 Se $\Gamma$ é consistente, então $\Gamma$ tem modelo.

Demonstração: De acordo com o Teorema de Lindenbaum, todo conjunto consistente $\Gamma$ pode ser estendido a um conjunto consistente maximal $\Delta$.

Então, verificamos que $\Delta$ tem um modelo e como $\Gamma \subseteq \Delta$, então o conjunto $\Gamma$ também tem modelo. 
Seja $E$ um conjunto unitário qualquer. Decorre que, $P(E)=\{\varnothing, E\}$ e que $\mathcal{F}=\{E\}$.

Definimos a sequinte valoração $v: \operatorname{For}(\mathrm{KD}) \rightarrow \mathcal{B}^{\mathcal{F}}$

$$
v(p)=E \Leftrightarrow p \in \Delta \text {. }
$$

Então, mostramos que para toda fórmula $\varphi, v(\varphi)=E \Leftrightarrow \varphi \in \Delta \mathrm{e}$, deste modo, $\left(\mathcal{B}^{\mathcal{F}}, v\right)$ é um $\mathrm{D}$-modelo para $\Delta$.

A demonstração é por indução sobre a complexidade de $\varphi$.

Se o operador principal de $\varphi$ é um operador Booleano, então o caso é simples e usual.

Agora, se $\varphi$ é do tipo $0 \psi$, então:

Se $v(O \psi)=E$, então $\mathcal{B}^{\mathcal{F}} \vDash O \psi \Leftrightarrow v(\psi) \in \mathcal{F}$. Daí, $v(\psi)=E$ e, pela hipótese de indução, $\psi \in \Delta$. Como $\Delta$ é uma teoria, então $\Delta \vdash 0 \psi$ e, portanto, $0 \psi \in \Delta$.

Se $v(O \psi) \neq E$, então $v(\psi)=\emptyset$ e, pela hipótese de indução, $\psi \notin \Delta$. Como $\Delta$ é uma teoria consistente e maximal, segue que $\Delta \vdash \neg \psi$. Pela regra Nec, $\Delta \vdash 0 \neg \psi$. Suponhamos agora que $0 \psi \in \Delta$, isto é, $\Delta \vdash 0 \psi$, por OC, teríamos que $\Delta \vdash \perp$ e $\Delta$ não seria teoria consistente. Logo, $\mathrm{O} \psi \notin \Delta$.

Desse modo, $\mathcal{B}^{\mathcal{F}}$ é um $\mathrm{D}$-modelo para $\Delta$.

Corolário 4.15 (Completude Forte) Se $\Gamma \vDash \varphi$, então $\Gamma \vdash \varphi$.

Demonstração: Se $\Gamma \vDash \varphi$, então todo modelo de $\Gamma$ é também um modelo de $\varphi$ e, deste modo, não há modelo de $\Gamma \cup\{\neg \varphi\}$. Então, do teorema anterior, $\Gamma \cup\{\neg \varphi\}$ é inconsistente e, pela Proposição 4.10, segue que $\Gamma \vdash \varphi$. -

\section{Considerações finais}

Ao olharmos para a terceira apresentação de KD, conforme surgiu neste artigo, nós pudemos reconhecer aspectos dos filtros na sua essência. Então, desenvolvemos os resultados seguintes, que mostraram que os filtros próprios são modelos adequados para este sistema deôntico básico.

Ao buscarmos na literatura sobre lógicas modais, não encontramos a vinculação do conceito de filtro como modelos para a lógica KD.

Por outro lado, como é bem conhecido que os usuais modelos de Kripke para KD são estruturas de Kripke relacionais, em que a relação de acessibilidade deve ser serial, então em próximos passos, desejamos investigar possíveis vínculos entre os modelos de filtros próprios e modelos de Kripke seriais para KD.

\section{Referências}

BELL, J. L.; MACHOVER, M. A course in mathematical logic. Amsterdam: NorthHolland, 1977.

BLACKBURN, P.; RIJKE, M.; VENEMA, Y. Modal logic. Cambridge: Cambridge University Press, 2001.

CARNIELLI, W. A.; PIZZI, C. Modalità e multimodalità. Milano: Franco Angeli, 2001. CHAGROV, A.; ZAKHARYASCHEV, M. Modal logic. Oxford: Clarendon Press, 1997. CHELLAS, B. Modal logic: an introduction. Cambridge: Cambridge University Press, 1980. 
DUNN, J. M.; HARDEGREE, G. M. Algebraic methods in philosophical logic. Oxford: Oxford University Press, 2001.

EBBINGHAUS, H. D.; FLUM, J.; THOMAS, W. Mathematical logic. New York: Springer-Verlag, 1984.

ENDERTON, H. B. A mathematical introduction to logic. San Diego: Academic Press, 1972.

FEITOSA, H. A.; PAULOVICH, L. Um prelúdio à lógica. São Paulo: Editora UNESP, 2005.

FITTING, M.; MENDELSOHN, R. L. First-order modal logic. Dordrecht: Kluwer, 1998.

MENDELSON, E. Introduction to mathematical logic. 3. ed. Monterey, CA: Wadsworth and Brooks / Cole Advanced Books and Software, 1987.

MIRAGLIA, F. Cálculo proposicional: uma interação da álgebra e da lógica. Campinas: UNICAMP/CLE, 1987. (Coleção CLE, v. 1).

RASIOWA, H.; SIKORSKI, R. The mathematics of metamathematics. 2. ed. Waszawa: PWN - Polish Scientific Publishers, 1968.

RASIOWA, H. An algebraic approach to non-classical logics. Amsterdam: NorthHolland, 1974.

\section{Sobre os autores}

Hércules de Araújo Feitosa

Doutor em Lógica e Filosofia da Ciência pela Universidade Estadual de Campinas - UNICAMP - IFCH (1998). Professor na UNESP, Faculdade de Ciências, Departamento de Matemática, Campus de Bauru e credenciado no Programa de Pós-Graduação em Filosofia da UNESP - FFC - Marília.

Email: hercules.feitosa@unesp.br

Marcelo Reicher Soares

Doutor em Matemática pela Universidade de São Paulo - USP (2000), Professor Assistente Doutor na Universidade Estadual Paulista Júlio de Mesquita Filho - UNESP e professor no Programa de PósGraduação em Matemática em Rede Nacional PROFMAT. E-mail: reicher.soares@unesp.br

\section{Cristiane Alexandra Lázaro}

Doutora em Matemática pela Universidade Estadual de Campinas (2008). Professora assistente doutora da Universidade Estadual Paulista Júlio de Mesquita Filho-UNESP.

E-mail: cristiane.lazaro@unesp.br

Recebido em 31/07/2019

Aprovado em 30/09/2019

\section{Como referenciar esse artigo}

FEITOSA, Hércules de Araújo; SOARES, Marcelo Reicher; LÁZARO, Cristiane Alexandra. Lógica deôntica básica e filtros. Argumentos: Revista de Filosofia. Fortaleza, ano 11, n. 22, p. 7-16, jul.-dez. 2019. 